\title{
Hair follicle transcriptome profiles during the transition from anagen to catagen in Cashmere goat (Capra hircus)
}

\author{
Y.X. Fan ${ }^{1 *}$, R.B. Wu' ${ }^{2 *}$ X. Qiao', Y.J. Zhang ${ }^{1}$, R.J. Wang ${ }^{1}$, R. Su${ }^{1}$, J.H. Wu ${ }^{3,4,6}$, \\ Y. Dong ${ }^{5,6}$ and J.Q. Li ${ }^{1}$ \\ ${ }^{1}$ College of Animal Science, Inner Mongolia Agricultural University, \\ Hohhot, Inner Mongolia, China \\ ${ }^{2}$ College of Basic Medical Sciences, Inner Mongolia Medical University, Hohhot, China \\ ${ }^{3}$ Inner Mongolia Prataculture Research Center, Chinese Academy of Science, \\ Hohhot, China \\ ${ }^{4}$ Animal Husbandry Institute, Inner Mongolia Academy of Agricultural \& Animal \\ Husbandry Sciences, Hohhot, China \\ ${ }^{5}$ Kunming University of Science and Technology, Kunming, China \\ ${ }^{6}$ State Key Laboratory of Genetic Resources and Evolution, \\ Kunming Institute of Zoology, Chinese Academy of Sciences, Kunming, China \\ *These authors contributed equally to this study. \\ Corresponding authors: J.H. Wu / J.Q. Li \\ E-mail: wujianghonglong@126.com / loyalyang@163.com
}

Genet. Mol. Res. 14 (4): 17904-17915 (2015)

Received August 11, 2015

Accepted October 2, 2015

Published December 22, 2015

DOI http://dx.doi.org/10.4238/2015.December.22.15

ABSTRACT. Previous molecular genetic studies of the goat hair life cycle have focused primarily on a limited number of genes and proteins. To identify additional genes that may play important roles in hair follicle cycle regulation, Illumina sequencing technology was used to catalog differential gene expression profiles in the hair growth cycle (anagen to catagen) of goat, comparing the primary hair follicle with the secondary hair follicle. There were 13,769 and 12,240 unigenes assembled from the reads obtained from primary hair follicle and secondary hair follicle, respectively. Genes encoding keratin proteins and keratin-associated proteins were the 
most highly expressed. A total of 5899 genes were differentially expressed in anagen vs catagen primary hair follicles, with 532 genes up-regulated and 5367 genes down-regulated. A total of 5208 genes were differentially expressed in anagen vs catagen secondary hair follicle, including 545 genes that were up-regulated and 4663 genes that were down-regulated. Numerous hair growth genes are expressed in the goat hair follicle, of which 73 genes showed co-up-regulation in both hair follicles during the anagen stage. Many of these up-regulated genes, such as STC2, VEGFR, and ROR2, are known to be transfactors in the process of cell differentiation and in the cell cycle. The differential gene expression profiles between primary hair follicles and secondary hair follicles obtained provide a foundation for future studies examining the network of gene expression controlling hair growth cycle in Cashmere goat.

Key words: Cashmere goat; Gene expression; Hair growth cycle; Transcriptome

\section{INTRODUCTION}

The Inner Mongolia Cashmere goat, a fiber double-coated (wool and cashmere) species famous for its luxurious fiber production trait, exhibits seasonal rhythms with a well-defined duration of fiber growth and is the world's largest breeding group (Allain and Renieri, 2010). The fleece of cashmere goats grows from specialized follicles in the skin. The fine undercoat is the source of cashmere fibers used for clothing, which is known as cashmere/down. The down is produced by secondary follicles and the wool/guard hair is produced by primary follicles (Ansari-Renani et al., 2011). There are many differences between the primary and secondary follicles regarding morphogenesis, cycle growth, and arrangement of the hair follicles. The timing of the cyclical phases differs for cashmere and hair fibers. The growing phase of hair occurs earlier than that of cashmere, and hair fibers as a group undergo a longer regressive period than cashmere (McDonald et al., 1987).

As a functional mini-organ, the hair follicle develops in an environment with dynamic and alternating changes of diverse molecular signals (Schneider et al., 2009). Generally, during catagen, hair growth generally ceases, which is accompanied by various obvious morphological alternations, such as dermal papilla condensation, moving upward, and many cytological changes, including epithelial cell and outer root sheath apoptosis (Krause and Foitzik, 2006). Several indications suggest that induction of primary and secondary hair follicle cycles may require different signaling pathways. As shown in mice with spontaneous mutation in the downless gene (which are characterized by lack of the primary hair follicle (PHF) and apparently normal development of the secondary hair follicle (SHF)), induction of PHF is dependent on signaling through the tumor necrosis factor receptor homolog, Edar (Headon and Overbeek, 1999).

For the Inner Mongolia Cashmere goat, the gene expression differences between primary hair follicles and secondary hair follicles from the anagen to catagen are unclear. Therefore, global measurements of gene expression and differential gene expression profiling of the different hair follicles should be conducted to advance the understanding of the molecular mechanisms underlying the anagen-to-catagen transition.

RNA-Seq technology is a high-throughput sequencing platform that allows for the detection of transcripts with low abundance, identification of novel transcript units, and determination of 
differential expression between different samples (Marguerat and Bähler, 2010; Qi et al., 2011). These new technologies can also provide accurate and digital gene expression profiles of sequenced transcripts (Bellin et al., 2009); in particular, they have been used to examine organisms for which limited genomic resources are available (Wang et al., 2009). To our knowledge, to date, RNASeq technology has not been used to analyze hair follicles in cashmere goats. In the present study, we deep-sequenced and then assembled the transcriptome of the PHF and SHF during the anagen and catagen stage phases using Illumina/Solexa sequencing technology. We then compared the digital and differential gene expression profiles between these two types of hair follicles during the transition from anagen to catagen.

\section{MATERIAL AND METHODS}

\section{Experimental animals and sample collection}

The Inner Mongolia White Cashmere goat is an outstanding breed because of its excellent cashmere production performance. Experimental Cashmere goat was obtained from Aerbasi White Cashmere Goat Breeding Farm in Inner Mongolia, China. The cashmere goats were raised by feeding practices according to the Cashmere goat standard. One adult individual (female, 2 years old) was randomly selected. Hair follicle samples were collected from the back of the goat from the anagen to catagen hair follicle developmental stages (anagen: October and catagen: January) (Figure 1).

a

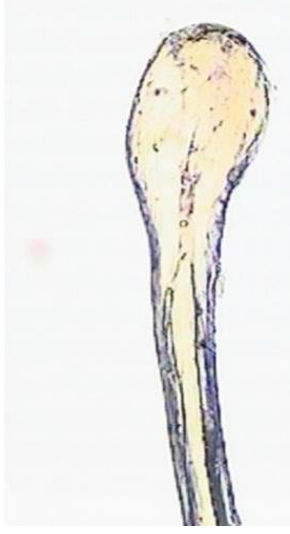

b

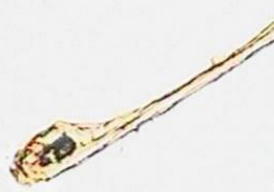

Figure 1. Representative examples of primary hair follicle (PHF) (a) and secondary hair follicle (SHF) (b). The SHF is much smaller than PHF, and cashmere (b) is much thinner than coat hair produced by PHFs (a) (100X magnification).

\section{Mapping reads to the reference genome}

Two databases were employed for sequence analysis, the goat (Capra hircus) reference gene (http://goat.kiz.ac.cn/GGD/download/CDS.tar.gz) and reference genomic DNA (http:// caprinae.kiz.ac.cn/GGD/download/Goat\%20genome\%20assembly.tar.gz). Sequencing-received raw image data were transformed by base culling into sequence data. Prior to mapping the reads to the reference database, we filtered all sequences to remove adaptor sequences and low-quality 
sequences (percentage of low-quality bases with a quality value $\leq 5$ was $>50 \%$ in a read). The remaining reads were aligned to the goat genome using SOAPaligner/soap2, allowing up to 2 base mismatches.

\section{Expression profiling and analysis of differential expression}

After filtering low-quality/contaminated/polymerase chain reaction artifact reads, reads from RNA-seq data of PHF and SHF paired samples were mapped against the goat assembly using Tophat (version 1.2.0, bowtie v0.12.5, parameters "-m 1 -mate-std-dev 20 -coverage-search -microexon-search"). The fragments per kilobase of exon per million fragments mapped (FPKM) was calculated for each protein-coding gene using Cuffdiff (http://cufflinks.cbcb.umd.edu, version 0.8.2). The significance level ( $P$ value) of the differential expressed genes between 2 samples was calculated using Cuffdiff with default parameters. All 4 sets of data were compared using the FPKM values. Genes with FPKM > 5 in each HF sample were used as a stringent cutoff to identify expressed genes, and FPKM > 0.1 in each HF were used as a loose cutoff to identify expressed genes. Differentially expressed genes were defined by at least a 2-fold FPKM change between PHF and SHF samples and showed the same direction of change in all 3 PHF/SHF comparisons.

\section{Analysis of gene ontology}

Hypergeometric tests were performed on annotations using updated databases from The Gene Ontology for GO categories from the Biological Process and Kyoto Encyclopedia of Genes and Genomes for pathways in ClueGO. The ClueGO output was restricted to GO categories with $q \leq 0.01$, and $\mathrm{GO}$ term fusion was used to avoid redundancy prior to visualization of networks in the Cytoscape environment. Grouping of terms was based on kappa statistics, and the leading term was highlighted in the networks in italics. To globally analyze epithelial populations, only GO levels from 1 to 12 were allowed, with at least 6 genes per node and a minimal representation of $5 \%$ genes for each term. Specific gene lists for global terms were then remapped for more detailed analysis. No filtering was performed when significant genes were mapped to the Kyoto Encyclopedia of Genes and Genomes (KEGG) pathways.

\section{RESULTS}

\section{Overview of the transcriptome in hair follicles}

To obtain comprehensive transcripts of goat hair follicles and to provide an overview of both the anagen and catagen gene expression profiles in the Cashmere goat, primary hair follicles during anagen in October (PHF-Oct), the primary hair follicle during catagen in January (PHF-Jan), and the secondary hair follicle during anagen (SHF-Oct) and catagen (SHF-Jan) from were sampled from the back of the goat. The hair follicles used in experiments were sampled from the same location on the back of goat. To obtain an overview of the transcription profile in the hair development cycle, we performed high-throughput RNA-seq utilizing paired-end Illumina sequencing technology on poly (A)-enriched RNAs isolated from PHF and SHF. Four independent RNA-Seq libraries were constructed and sequenced using Illumina/Solexa technology (Table 1). The generated reads were then aligned to the goat genome (http://caprinae.kiz.ac.cn/GGD/) using 3 aligners, Burrows-Wheeler Alignment (Li and Durbin, 2009), Bowtie (Langmead et al., 2009), and TopHat (Trapnell et al., 2009), and the combined results were further analyzed. 
Table 1. Read numbers for the 4 independent hair follicle libraries.

\begin{tabular}{lcc}
\hline Library name & Total length (bp) & Clean reads \\
\hline SHF-Oct & 2949246900 & 16384705 \\
SHF-Jan & 2596684860 & 14426027 \\
PHF-Oct & 3244219920 & 18023444 \\
PHF-Jan & 4297624020 & 23875689 \\
\hline
\end{tabular}

Secondary hair follicle (SHF), primary hair follicle (PHF).

\section{Evaluation of differential expression genes (DEGs)}

After mapping the sequencing reads to the reference genome with TopHat, transcripts were assembled and their relative expression levels were calculated using Cufflinks in FPKM. Next, the sub-program, Cuffdiff was used to calculate the differential expression. Cufflinks calculates the differential gene expression as the ratio of the anagen to the catagen for every gene as well as the statistical significance of the values. We used 4 samples form 2 different follicle stages (anagen and catagen), including 2 paired samples (primary hair follicles and secondary hair follicles during the same month, anagen and catagen) form one individual for comparative transcriptome analysis. Approximately 14,221 genes were detected in both secondary hair follicles and primary hair follicles (Figure 2). The samples were classified to 4 paired sample sets to compare the expression level (based on RPKM values) between the 2 types of follicles within each sample set. We identified 9345 genes with comparable expression levels shared between both sets of hair follicles. The down- and up-regulated gens within each sample set were intersected as candidates.

To analyze the expression patterns of genes showing conserved expression between PHF and SHF, we performed hierarchical clustering to group the genes according to similarities in their patterns of gene expression. Two-dimensional hierarchical clustering classified 9345 DEGs into several expression cluster groups (Figure 3) based on the similarity of their expression patterns, representing the number of profiles indicated using figure of merit analysis. Visual inspection of these expression groups suggested diverse and complex patterns of regulation. These clusters contained genes that were positively or negatively modulated over the entire time course, whereas genes expressed during the 2 stages of hair follicle development showed some differences. There were clusters with relatively minor differences between PHF-Oct vs PHF-Jan and SHF-Oct vs SHFJan, but marked differences with catagen vs telogen.

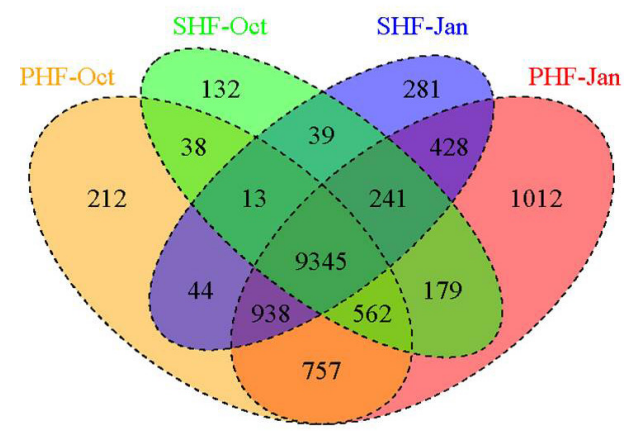

Figure 2. All transcripts expressed in PHFs during anagen at October (PHF-Oct), PHFs during catagen at January (PHFJan), and SHFs during anagen (SHF-Oct) and catagen (SHF-Jan). Gene expression results from the transcriptomes were analyzed using FPKM. PHF = primary hair follicle; SHF = secondary hair follicle. 


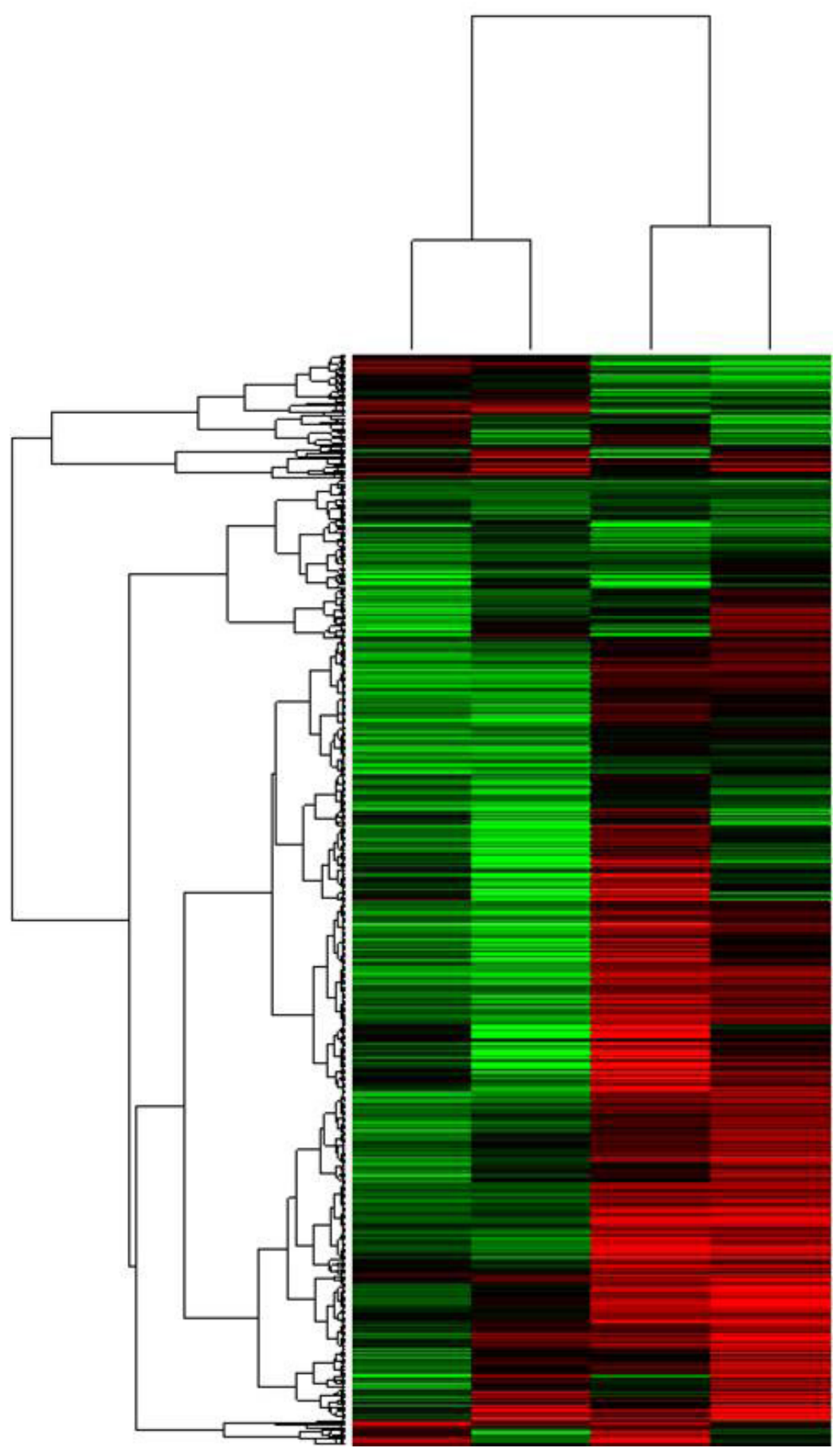

Figure 3. Hierarchical cluster analysis of gene expression based on log ratio RPKM data. The red color represents up-regulated genes and the green color represents down-regulated genes. 


\section{DEGs of PHF between anagen and catagen}

There were 532 upregulated and 5367 downregulated genes in PHF-Oct showing at least a 2-fold change over the PHF-Jan (Table 2). Stanniocalcin-2 (STC2) and YS037 were the top 2 most upregulated genes in PHF-Oct vs PHF-Jan, respectively. STC2 was upregulated by 153-fold, while YS037 was upregulated by 37 -fold. Among the top upregulated genes, runt-related transcription factor 2, vascular endothelial growth factor A (VEGFA), and inhibin beta A chain, both of which are associated with hair follicles development and growth, were upregulated by 13-, 9-, and 13-fold, respectively. Frizzled-8 and tyrosine-protein kinase transmembrane receptor ROR2, which is involved in the Wnt signaling pathway, was upregulated by 8- and 7-fold, respectively. CP17A and SNX17 were the 2 most downregulated genes in PHF-Oct vs PHF-Jan (127- and 68-fold, respectively).

Expression was determined using CuffDiff after Benjamini-Hochberg correction. The foldchange is the ratio of PHF-Oct FPKM to PHF-Jan FPKM.

Table 2. Gene expression summary of primary hair follicle (PHF).

\begin{tabular}{lcc}
\hline & & PHF-Oct \\
\hline Total genes expressed & 11,908 & 13,461 \\
PHF-Oct only & 307 & 1860 \\
PHF-Jan only & 532 & \\
Up-regulated (2-fold or greater difference) PHF-Oct vs PHF-Jan & 5367 & \\
Down-regulated (2-fold or greater difference) PHF-Oct vs PHF-Jan & & \\
\hline
\end{tabular}

\section{DEGs of SHF between anagen and catagen}

There were 10,549 expressed genes in SHF-Oct and 11,329 expressed genes in SHF-Jan, which were aligned to the reference genome (Table 2). There were 911 genes whose expression was only detected in SHF-Oct and 1691 genes whose expression was only detected in SHFJan. The differentially expressed genes in SHF-Oct compared to SHF-Jan showing statistically significant fold-changes ranged from 216.32 to 94.23 . There were 545 upregulated and 4663 downregulated genes in SHF-Oct showing an at least 2-fold change over SHF-Jan (Table 3).

Expression was determined using CuffDiff after Benjamini-Hochberg correction. The foldchange is the ratio of SHF-Oct FPKM to SHF-Jan FPKM.

Table 3. Gene expression summary of secondary hair follicle (SHF).

\begin{tabular}{lcc}
\hline & & SHF-Oct \\
\hline Total genes expressed & 10,549 & 11,329 \\
SHF-Oct only & 911 & 1691 \\
SHF-Jan only & 545 & 4663 \\
Up-regulated (2-fold or greater difference) SHF-Oct vs SHF-Jan & 4663 \\
Down-regulated (2-fold or greater difference) SHF-Oct vs SHF-Jan &
\end{tabular}

\section{Gene ontology enrichment analysis of PHF and SHF during the transition from anagen to catagen stage}

To evaluate the validity of this categorization, we performed Gene Ontology (GO) 
enrichment analysis of differentially expressed genes. This analysis was based on statistical significant overrepresentation of categories in the ontology Biological Process. The GO analysis for PHFs revealed the presence of functions related to the regulation of cellular process and cell cycle within the PHF. The term regulation of cellular process was identified as a group leading term (Figure 4). A bar chart of significantly enriched terms is available as supplemental information (Figure S1). In particular, we identified several processes central to hair follicle development, such as the Wnt receptor signaling pathway, cell cycle process, regulation of cell differentiation, and intracellular signal transduction. The time at which the hair follicle stops growing appears to be important, as the category apoptotic signaling pathway was significantly enriched.

These DEGs of PHF between anagen and catagen also mapped to known pathways according to KEGG analysis. Many pathways involve the signaling pathways; particularly, transforming growth factor-beta signaling and Notch signaling play important roles in hair follicle growth and development.

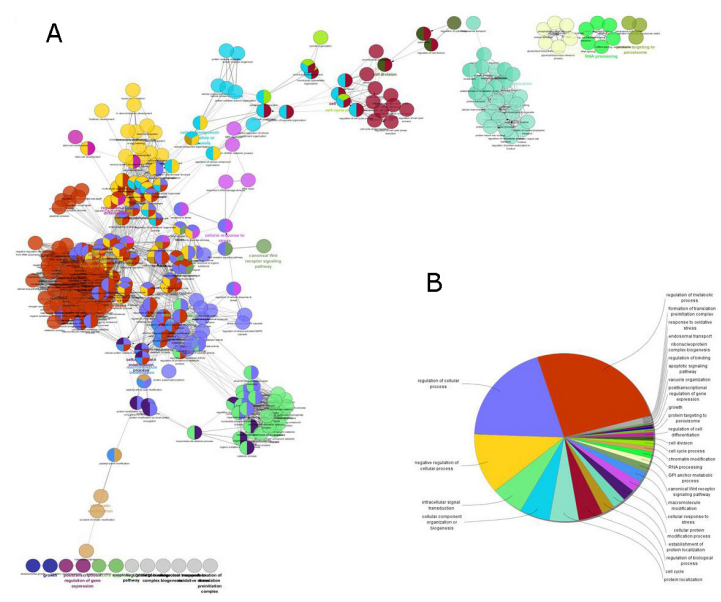

Figure 4. Gene ontology analysis of significantly differentially expressed genes in PHF. A. Network of significantly enriched terms. The color gradient shows the gene proportion of each cluster associated with the term. Equal proportions of the 2 clusters are represented in white. B. Functional groups in ClueGO Overview.

GO analysis for SHFs revealed the presence of functions indicative of the regulation of development process, regulation of cell motility, and cell cycle within the SHF. This is supported by the fact that the term regulation of cellular process was identified as a group leading term (Figure 5). A bar chart of significantly enriched terms is available as supplemental information (Figure S2). In particular, we identified several processes central to hair follicle cycle development, such as stem cell maintenance/differentiation/development, cell cycle process, cell cycle, regulation of cell cycle, mitotic cell cycle, and organic cyclic compound catabolic process. The point at the beginning on the secondary hair follicle response to hormones appears to be important, as the category intracellular steroid hormone receptor signaling pathway was significantly enriched (Figure 6).

These DEGs of SHF between anagen and catagen also mapped to known pathways according to KEGG. Many pathways involve signaling pathways; particularly, Wnt signaling, ErbB, and Notch signaling play important roles in hair follicle growth and development (Figure 7). These results of pathway analysis were similar to those of PHF assessment, as it helps both hair follicle types. The comparison of KEGG of 2 DGE clusters are displayed in charts showing the groups and their leading term as well as detailed term histograms for both cluster-specific and common terms (Figure 8). 


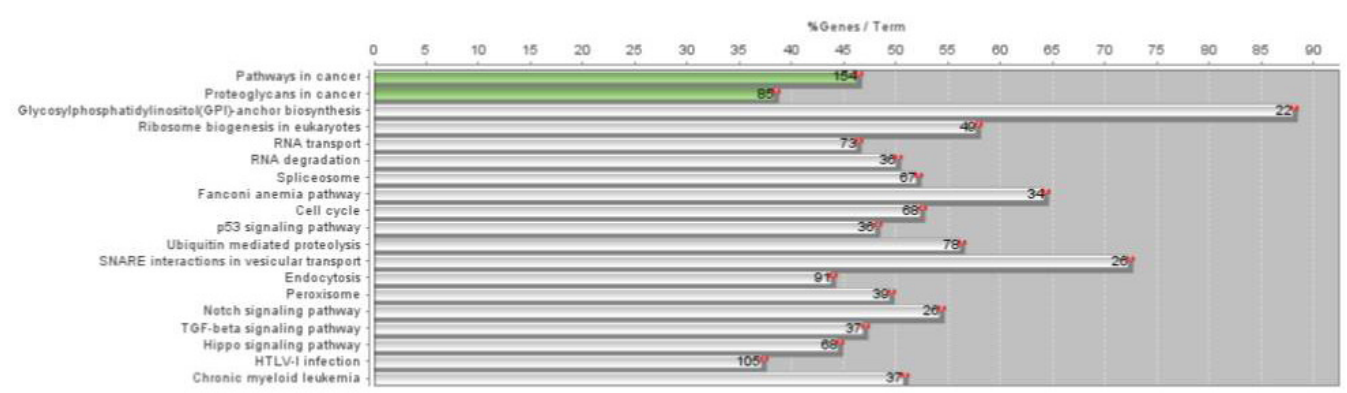

Figure 5. Pathway analysis of differentially expressed genes in PHF genes during the transition from anagen to catagen.
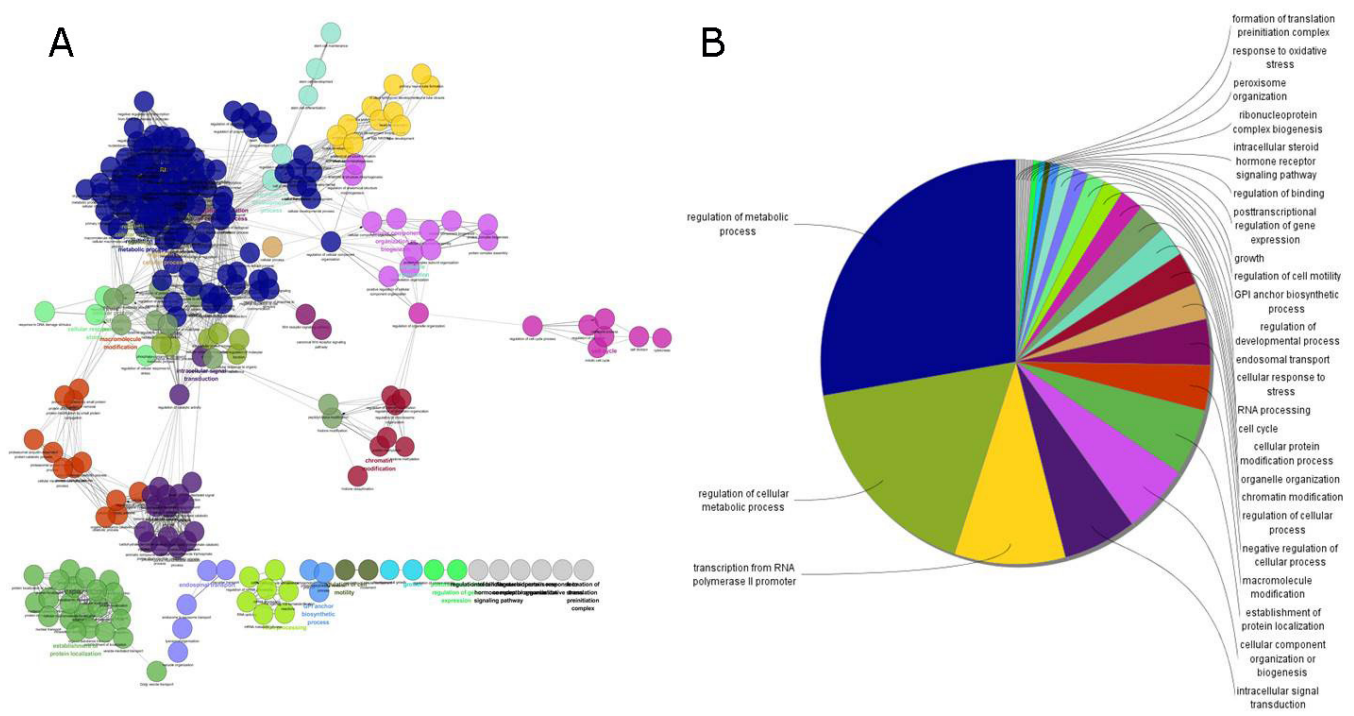

Figure 6. Gene ontology analysis of significantly differentially expressed genes in SHFs. A. Network of significantly enriched terms. The color gradient shows the gene proportion of each cluster associated with the term. Equal proportions of the 2 clusters are represented in white. B. Functional groups in ClueGO Overview.

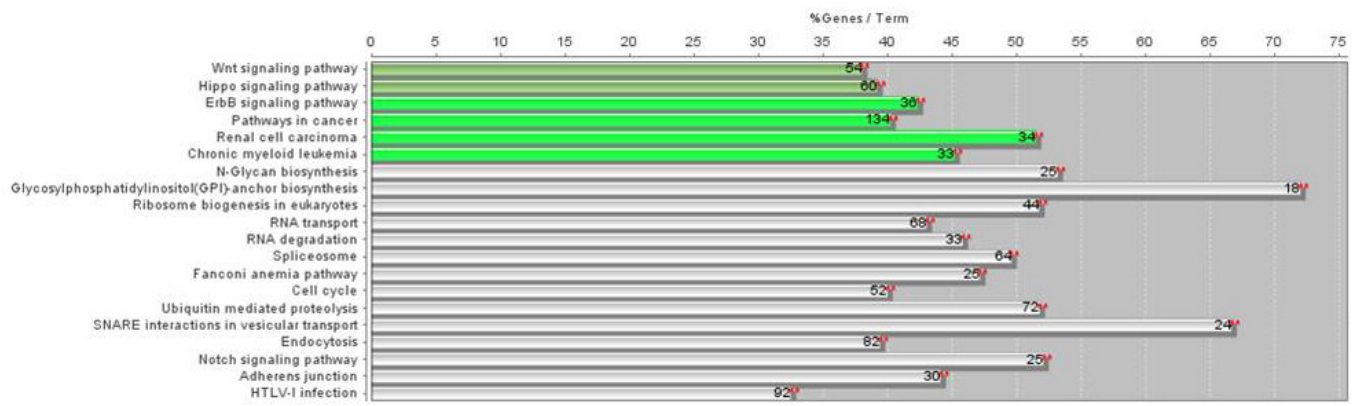

Figure 7. Pathway analysis of differentially expressed genes in PHF genes during the transition from anagen to catagen. 


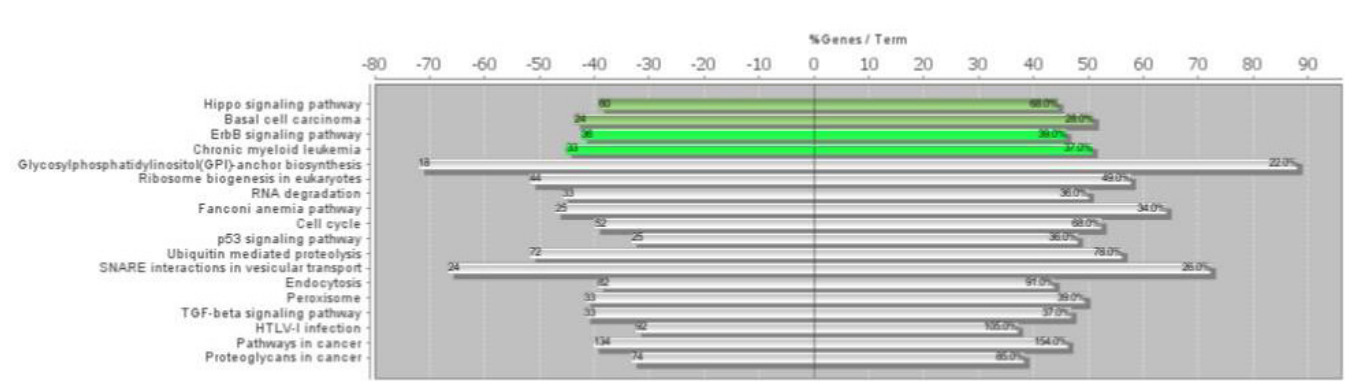

Figure 8. Comparison of KEGG for differentially expressed genes in PHF and SHF during the transition from anagen to catagen.

\section{DISCUSSION}

In this study, we sampled the hair follicle substituted for traditional skin tissue to more closely resemble the true hair cycle mechanism. Our study offers insights into the gene expression profiles of 2 hair follicle types during the anagen-catagen transition phase in cashmere goats. A limitation of our study was that the goat genomic annotation is not complete. To systematically study the difference between cashmere and hair development, we carried out transcriptome sequencing for the SHF and PHF from Inner Mongolia Cashmere goat, which is a major breed of Cashmere goat in China.

A comparative expression profiling strategy between different developmental stages was used to identify the subset of genes that were differently expressed. We first determined how hair follicle development differs in gene expression between the anagen to catagen stages. Some potential regulators of hair follicle development have been identified. First, the number of special expression genes between 2 hair follicle types of the 2 stages showed significant differences, reflecting a cumulative effect on phase traits. Most of these genes were commonly expressed in the PHF and SHF of the 2 stages, indicating the complexity of the sharing effect. Second, expression patterns, GO categories, and KEGG pathways associated with DEGs between the 2 developmental stages showed considerable differences. The KEGG for differentially expressed genes in PHF and SHF shared many gene pathways during the transition from anagen to catagen. This unique pattern between anagen and catagen indicates that switches in phase-related events occur on the physiological or molecular level during the transition.

The hair follicle is one of the defining features of mammalian species. The prerequisite for hair follicle development and cycling is the ability to switch the molecular signaling pathway on and off. These transformations are controlled by changes in the local signaling niche based on changes in the expression and activity levels of transcription factors, hormones, cytokines, neurotrophic factors, and their receptors that are recognized as key mediators of hair follicle cycling.

Among the differentially expressed hair follicle genes, STC2 showed the greatest level of differential expression in anagen vs catagen goat hair follicles. Mammalian STC2 are glycoprotein hormones identified as calcium/phosphate-regulating hormones (Zeiger et al., 2011). STC2 homozygous null mice are $10-15 \%$ larger and grow at a faster rate than wild-type control mice (Takei et al., 2012). In this study, the STC2 gene was significantly down-regulated in both types of hair follicle during catagen. Therefore, whether the expression pattern of these genes is related to developmental events of the hair follicle remains unknown. Further studies are necessary to confirm the roles of these co-regulated genes in Cashmere goat hair follicle development and cycling.

Genes in the 'regulation of morphogenesis of a branching structure)' ontology category, 
such as VEGFA, a member of the platelet-derived growth factor/VEGF family (Vincenti et al., 1996). VEGF expression is upregulated in the hyperplastic epidermis of psoriasis (Detmar et al., 1994) and in other skin diseases characterized by enhanced angiogenesis (Brown et al., 1995), and targeted overexpression of VEGF in the epidermis of transgenic mice resulted in enhanced skin vascularization with increased numbers of tortuous and leaky blood vessels (Detmar et al., 1998). Transgenic overexpression of VEGF in outer root sheath keratinocytes resulted in enhanced perifollicular vascularization, accelerated hair regrowth after depilation, and increased size of vibrissa follicles, hair follicles, and hair shafts (Yano et al., 2001). In this study, we found that the expression level of the VEGFA gene was significantly higher in PHF than in SHF, and the expression level of the VEGFA gene was significantly higher at anagen than at catagen in the same hair follicle. These results suggest that the expression level of the VEGFA gene is related to different sizes between PHF and SHF. Furthermore, the tyrosine-protein kinase transmembrane receptor ROR2 gene in the 'regulation of morphogenesis of a branching structure)' ontology category, also known as neurotrophic tyrosine kinase (Schwarzer et al., 2009), may be involved in the early formation of chondrocytes and may be required for cartilage and growth plate development (Stricker and Mundlos, 2011). In this study, the ROR2 gene was significantly downregulated in both types of hair follicle at catagen.

In conclusion, although the genes controlling hair follicle development and cycling in Cashmere goats remain largely unknown, in this study we identified a set differentially expressed known and novel genes in hair follicles potentially related to hair cycling growth and other physiological functions. The identified DEGs and pathways can be utilized to facilitate further studies of the detailed molecular mechanisms and provide a foundation for future studies to potentially manipulate cashmere traits via pharmacological and genetic approaches.

\section{ACKNOWLEDGMENTS}

Research supported by the National "863" Project (\#2013AA102506), the National natural Science Foundation of China (\#31201773, \#31260539, \#31272421), and the Excellent Young Scientist Foundation of Inner Mongolia Agricultural University of China (\#2014XYQ-1). Also, this research was supported by the Natural Science Foundation of Inner Mongolia (\#2013MS0414), the Youth Innovation Foundation of Inner Mongolia Academy of Agricultural \& Animal Husbandry Sciences (\#2013QNJJM03), and the State Key Laboratory of Genetic Resources and Evolution, Kunming Institute of Zoology, Chinese Academy of Sciences (\#GREKF13-02).

\section{Supplementary material}

\section{REFERENCES}

Allain D and Renieri C (2010). Genetics of fibre production and fleece characteristics in small ruminants, Angora rabbit and South American camelids. Animal 4: 1472-1481.

Ansari-Renani H, Ebadi Z, Moradi S, Baghershah HR, et al. (2011). Determination of hair follicle characteristics, density and activity of Iranian cashmere goat breeds. Small Ruminant Res. 95: 128-132.

Bellin D, Ferrarini A, Chimento A, Kaiser O, et al. (2009). Combining next-generation pyrosequencing with microarray for large scale expression analysis in non-model species. BMC Genomics 10: 555.

Brown LF, Olbricht SM, Berse B, Jackman RW, et al. (1995). Overexpression of vascular permeability factor (VPF/VEGF) and its endothelial cell receptors in delayed hypersensitivity skin reactions. J. Immunol. 154: 2801-2807.

Detmar M, Brown LF, Claffey KP, Yeo KT, et al. (1994). Overexpression of vascular permeability factor/vascular endothelial growth factor and its receptors in psoriasis. J. Exp. Med. 180: 1141-1146. 
Detmar M, Brown LF, Schön MP, Elicker BM, et al. (1998). Increased microvascular density and enhanced leukocyte rolling and adhesion in the skin of VEGF transgenic mice. J. Invest. Dermatol. 111: 1-6.

Headon DJ and Overbeek PA (1999). Involvement of a novel Tnf receptor homologue in hair follicle induction. Nat. Genet. 22: 370-374.

Krause K and Foitzik K (2006). Biology of the hair follicle: the basics. Semin. Cutan. Med. Surg. 25: 2-10.

Marguerat S and Bähler J (2010). RNA-seq: from technology to biology. Cell Mol. Life Sci. 67: 569-579.

McDonald B, Hoey W and Hopkins P (1987). Cyclical fleece growth in cashmere goats. Crop Pasture Sci. 38: 597-609.

Qi YX, Liu YB and Rong WH (2011). RNA-Seq and its applications: a new technology for transcriptomics. Yi Chuan 33: 1191-1202.

Schneider MR, Schmidt-Ullrich R and Paus R (2009). The hair follicle as a dynamic miniorgan. Curr. Biol. 19: 132-142.

Schwarzer W, Witte F, Rajab A, Mundlos S, et al. (2009). A gradient of ROR2 protein stability and membrane localization confers brachydactyly type B or Robinow syndrome phenotypes. Hum. Mol. Genet. 18: 4013-4021.

Stricker S and Mundlos S (2011). FGF and ROR2 receptor tyrosine kinase signaling in human skeletal development. Curr. Top. Dev. Biol. 97: 179-206.

Takei Y, Yamamoto H, Sato T, Otani A, et al. (2012). Stanniocalcin 2 is associated with ectopic calcification in a-klotho mutant mice and inhibits hyperphosphatemia-induced calcification in aortic vascular smooth muscle cells. Bone 50: 998-1005.

Vincenti V, Cassano C, Rocchi M and Persico G (1996). Assignment of the vascular endothelial growth factor gene to human chromosome 6p21.3. Circulation 93: 1493-1495.

Wang Z, Gerstein M and Snyder M (2009). RNA-Seq: a revolutionary tool for transcriptomics. Nat. Rev. Genet. 10: 57-63.

Yano K, Brown LF and Detmar M (2001). Control of hair growth and follicle size by VEGF-mediated angiogenesis. J. Clin. Invest. 107: 409-417.

Zeiger W, Ito D, Swetlik C, Oh-hora M, et al. (2011). Stanniocalcin 2 is a negative modulator of store-operated calcium entry. Mol. Cell. Biol. 31: 3710-3722. 